as those who are at the front end of manufacturing and maintaining the operations at the factory. And it has been a successful enterprise, because not only did we achieve certification, but also because we are maintaining and extending the quality initiative with the support of everyone. There is no doubt that getting ISO 9002 has given us an achievement in which we have all been able to share and of which we are all proud. It is this that has given us the encouragement to venture into other activities associated with improving the quality of our operations.

As far as the future is concerned we have plans to build on the foundation that has been created by obtaining ISO certification. We want to move on from here from this foundation and develop a number of other quality initiatives that will lead us along the road to being a total quality managed site. The things that we want to do in the future involve consolidating everything that we have put into the quality system so that we continue to benefit from all that the system has to offer and the involvement of people.

We want to apply Statistical Process Control to all of our production processes and have already embarked on this in one of our production units. We intend to extend this in the months to come to the other units as well. For this purpose a large number of our chemists are undergoing training in the techniques associated with SPC.

We are also developing specific projects which might be classed under the heading of Continuous Improvement. These are associated with better performance, increased efficiency, better yield so that all across the site there might be a general raising of the quality of our operations.

We also want to develop systems whereby we can gather together all the costs of non-conformance not just in production areas but in all areas of the factory so that we might eliminate the waste of time, materials and money. Not only is this an important measure of the quality of operations at a factory, but it should also indicate that Quality is cost effective and can be used to make a positive contribution to profits. This at the end of the day is why we are in business isn't it?
Chimia 46 (1992) 277-281

(C) Neue Schweizerische Chemische Gesellschaft ISSN 0009-4293

\title{
Vers la qualité totale: évolution ou revolution
}

\author{
Pierre Manhes*
}

\section{Introduction générale}

Au moment de l'élaboration de l'encyclopédie, et avant les profonds bouleversements de la fin du 18ème siècle, $M$. le Marquis de Pompignan dans le discours préliminaire à ses poésies sacrées, reprenait la querelle des anciens et des modernes et proférait en 1788:

'Quoiqu'en disent les plaisants du siècle, il vaut mieux ennuyer son prochain que le corrompre ou le pervertir'. Le reste de l'ouvrage, d'ailleurs, montre bien qu'en tout cas ce marquis-là ne pervertit personne.

J'ai bien peur de ne pas suivre l'exemple de ce sage et de réussir pour ma part l'exploit de corrompre mon prochain tout en l'ennuyant.

Mon propos sera d'essayer de clarifier ce que signifie la notion de qualité pour notre entreprise et comment elle y est mise en œuvre, à travers mes expériences, et donc ma perception de l'action de Nestlé dans ce domaine. Les discours d'anciens combattants sont souvent ennuyeux et l'ob-

\footnotetext{
*Correspondance: P. Manhes

Nestec SA

Nestlé

$\mathrm{CH}-1800$ Vevey
}

jectivité n'est généralement pas leur fort. Merci donc de vos efforts à me suivre.

\section{Introduction}

Et d'abord, qu'est-ce que la qualité totale? Chaque époque amène son lot de sigles et d'expressions nouvelles qu'il est de bon ton d'utiliser pour paraître 'dans le vent'. Pourquoi n'en serait-il pas de même avec ce concept, et les esprits forts ne feraient-ils pas mieux d'attendre la naissance de l'expression suivante pour se gausser de ceux qui, à corps et à cris, se sont lancés à l'assaut de cet autre mirage organisationnel, nouvelle terre promise de managers romantiques et en tout cas source de profit des cabinets de conseils.

Je n'ai pas de définition originale mais quelques remarques s'appuyant plus particulièrement sur notre expérience:

- tout d'abord une définition est souvent une déclaration d'intention, et, comme dans une auberge espagnole, on trouve dans le concept de la qualité totale ce qu'on y apporte: qualité du produit, du service au client, définition du produit en fonction des besoins de la clientèle, amélioration de la productivité, flux tendu, et j'en passe. Les définitions varient d'une entreprise à l'autre, d'une circonstance à l'autre, et il faut distin-

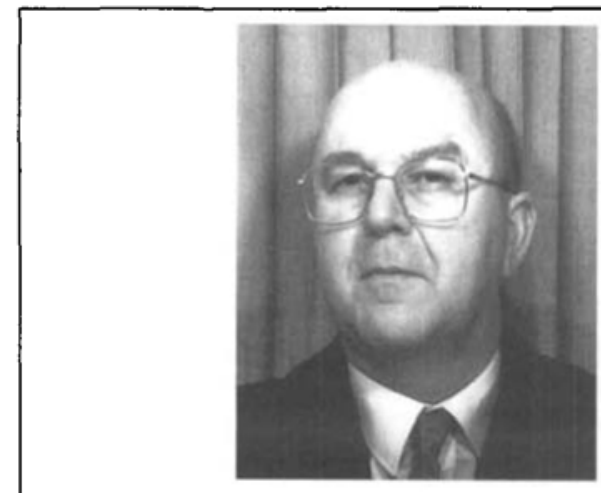

Pierre Manhes. Ingénieur Chimiste EFTLyon, France. Carrière professionnelle chez Nestlé, d'abord dans le cadre de Quality Control au Venezuela, puis dans des laboratoires régionaux en Colombie et en Afrique du Sud, puis direction d'usine en Afrique du Sud et en Colombie. Il fut par la suite Directeur Technique au Danemark. Depuis fin 1990 il est Assistant du Coordonateur Technique de la Zone 1 (Europe).

guer le permanent et fondamental du circonstanciel.

- Ensuite, pour beaucoup d'entre nous, en partie à cause de ce qui précède, il ne nous est pas apparu au premier abord que le concept fut bien nouveau. Dignes émules de M.Jourdain, nous avions fait sans le savoir de la qualité totale depuis fort longtemps. En effet, plusieurs aspects de la culture de l'entreprise sont parties intégrantes, se redéfinissent, et se renforcent dans le concept de qualité totale.

- Par ailleurs, un nouveau concept n'élimine pas l'antérieur. Je ne considère pas que dans un édifice les fondations sont moins nécessaires que les étages supérieurs. Bien au contraire, je me propose dans ce qui suit d'expliquer le développement du concept de qualité dans notre entreprise étape par étape. 
as those who are at the front end of manufacturing and maintaining the operations at the factory. And it has been a successful enterprise, because not only did we achieve certification, but also because we are maintaining and extending the quality initiative with the support of everyone. There is no doubt that getting ISO 9002 has given us an achievement in which we have all been able to share and of which we are all proud. It is this that has given us the encouragement to venture into other activities associated with improving the quality of our operations.

As far as the future is concerned we have plans to build on the foundation that has been created by obtaining ISO certification. We want to move on from here from this foundation and develop a number of other quality initiatives that will lead us along the road to being a total quality managed site. The things that we want to do in the future involve consolidating everything that we have put into the quality system so that we continue to benefit from all that the system has to offer and the involvement of people.

We want to apply Statistical Process Control to all of our production processes and have already embarked on this in one of our production units. We intend to extend this in the months to come to the other units as well. For this purpose a large number of our chemists are undergoing training in the techniques associated with SPC.

We are also developing specific projects which might be classed under the heading of Continuous Improvement. These are associated with better performance, increased efficiency, better yield so that all across the site there might be a general raising of the quality of our operations.

We also want to develop systems whereby we can gather together all the costs of non-conformance not just in production areas but in all areas of the factory so that we might eliminate the waste of time, materials and money. Not only is this an important measure of the quality of operations at a factory, but it should also indicate that Quality is cost effective and can be used to make a positive contribution to profits. This at the end of the day is why we are in business isn't it?
Chimia 46 (1992) 277-281

(C) Neue Schweizerische Chemische Gesellschaft ISSN 0009-4293

\section{Vers la qualité totale: évolution ou revolution}

\author{
Pierre Manhes*
}

\section{Introduction générale}

Au moment de l'élaboration de l'encyclopédie, et avant les profonds bouleversements de la fin du 18ème siècle, $M$. le Marquis de Pompignan dans le discours préliminaire à ses poésies sacrées, reprenait la querelle des anciens et des modernes et proférait en 1788:

'Quoiqu'en disent les plaisants du siècle, il vaut mieux ennuyer son prochain que le corrompre ou le pervertir'. Le reste de l'ouvrage, d'ailleurs, montre bien qu'en tout cas ce marquis-là ne pervertit personne.

J'ai bien peur de ne pas suivre l'exemple de ce sage et de réussir pour ma part l'exploit de corrompre mon prochain tout en l'ennuyant.

Mon propos sera d'essayer de clarifier ce que signifie la notion de qualité pour notre entreprise et comment elle y est mise en œuvre, à travers mes expériences, et donc ma perception de l'action de Nestlé dans ce domaine. Les discours d'anciens combattants sont souvent ennuyeux et l'ob-

\footnotetext{
*Correspondance: P. Manhes

Nestec SA

Nestlé

$\mathrm{CH}-1800$ Vevey
}

jectivité n'est généralement pas leur fort. Merci donc de vos efforts à me suivre.

\section{Introduction}

Et d'abord, qu'est-ce que la qualité totale? Chaque époque amène son lot de sigles et d'expressions nouvelles qu'il est de bon ton d'utiliser pour paraître 'dans le vent'. Pourquoi n'en serait-il pas de même avec ce concept, et les esprits forts ne feraient-ils pas mieux d'attendre la naissance de l'expression suivante pour se gausser de ceux qui, à corps et à cris, se sont lancés à l'assaut de cet autre mirage organisationnel, nouvelle terre promise de managers romantiques et en tout cas source de profit des cabinets de conseils.

Je n'ai pas de définition originale mais quelques remarques s'appuyant plus particulièrement sur notre expérience:

- tout d'abord une définition est souvent une déclaration d'intention, et, comme dans une auberge espagnole, on trouve dans le concept de la qualité totale ce qu'on y apporte: qualité du produit, du service au client, définition du produit en fonction des besoins de la clientèle, amélioration de la productivité, flux tendu, et j'en passe. Les définitions varient d'une entreprise à l'autre, d'une circonstance à l'autre, et il faut distin-

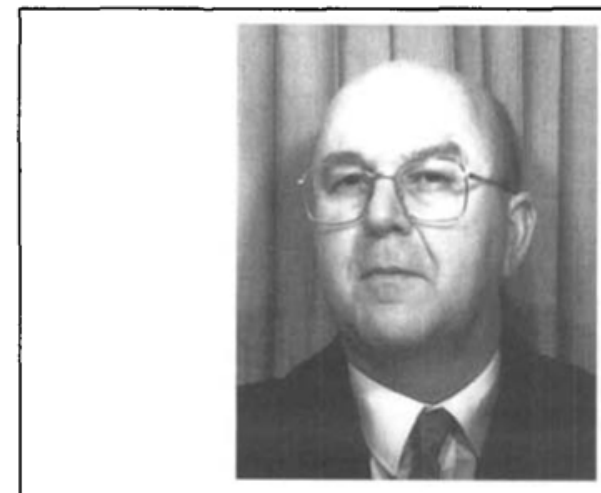

Pierre Manhes. Ingénieur Chimiste EFTLyon, France. Carrière professionnelle chez Nestlé, d'abord dans le cadre de Quality Control au Venezuela, puis dans des laboratoires régionaux en Colombie et en Afrique du Sud, puis direction d'usine en Afrique du Sud et en Colombie. Il fut par la suite Directeur Technique au Danemark. Depuis fin 1990 il est Assistant du Coordonateur Technique de la Zone 1 (Europe).

guer le permanent et fondamental du circonstanciel.

- Ensuite, pour beaucoup d'entre nous, en partie à cause de ce qui précède, il ne nous est pas apparu au premier abord que le concept fut bien nouveau. Dignes émules de M.Jourdain, nous avions fait sans le savoir de la qualité totale depuis fort longtemps. En effet, plusieurs aspects de la culture de l'entreprise sont parties intégrantes, se redéfinissent, et se renforcent dans le concept de qualité totale.

- Par ailleurs, un nouveau concept n'élimine pas l'antérieur. Je ne considère pas que dans un édifice les fondations sont moins nécessaires que les étages supérieurs. Bien au contraire, je me propose dans ce qui suit d'expliquer le développement du concept de qualité dans notre entreprise étape par étape. 
La qualité totale vise l'engagement de tous pour la mobilisation optimale des ressources de l'entreprise afin de satisfaire les besoins de la clientèle. Tous les secteurs de l'entreprise sont concernés, la mobilisation est permanente pour une recherche constante des améliorations possibles.

Il s'agit d'une stratégie globale, pas d'une panacée, et nous aurons l'occasion de revenir sur ce point plus en avant dans notre exposé.

Par ailleurs, l'environnement, c'est-àdire les conditions générales, l'atmosphère, les modes de travail, les communications prévalant dans l'entreprise constituent des facteurs essentiels à prendre en compte avant de se lancer dans un programme de qualité totale.

\section{Evolution}

\section{La culture de Nestlé}

Qu'en est-il donc pour notre entreprise? Nestlé existe maintenant depuis 125 ans et sa culture très forte, quoique peu explicitée, constitue le cadre qui a conduit naturellement au développement d'une stratégie de qualité. Notre entreprise multinationale continue à développer, par la formation et l'échange des personnels, une culture propre, vivante et dynamique, qui se traduit par une même manière de faire les choses, des non-écrits lourds de sens, des valeurs communes. Un trait particulier de la culture de Nestlé est qu'elle est suisse. Il serait malvenu qu'un Français détaille devant cet auditoire les caractéristiques de la culture suisse. Cependant, certains traits, peut-être plus perceptibles à l'étranger parce que moins naturellement vécus, peuvent être relevés, qui sont partie de notre philosophie. Je n'en citerai que quelques-uns.

a) L'opiniâtreté et le goût de l'effort: il peut paraître difficile de donner le meilleur de soi-même dans des conditions de vie délicates. En fait, c'est le contraire que nous avons tous connus.

La formation plus rapide et plus solide d'un groupe de vie et de travail, l'exaltation quasi sportive devant la tâche à accomplir, l'opportunité donnée très tôt d'exercer des responsabilités, sont des facteurs que tout cadre international de Nestlé a vécus.

Lorsque j'étais en Colombie, c'était l'époque où Lucho Herrera faisait des prouesses dans le Tour de France: on ne parlait plus que de cela dans tout le pays. Je disais 'le champion ne l'est pas tant quand il monte sur le podium mais quand il sue et se dépasse dans la montée des cols'. b) L'obsession du travail bien fait et la modestie: ce que l'un de mes chefs traduit par: être en permanence positivement insatisfait. Dans une fabrique où je me trouvais, nous avions établi, avec l'aide d'un consultant la notion du travail complet (en anglais le 'complete work') à l'aide d'un test très simple: 'Si vous étiez votre propre patron, seriez-vous disposé à signer le document que vous avez préparé et mettre tout le poids de votre stature professionnelle à soutenir son contenu?'.

c) La simplicité dans les communications, ce que $\mathrm{j}$ 'appellerai une longue tradition de démocratie, donc de respect mutuel. Ce trait, particulièrement sensible pour le Français que je suis, supprime la distance entre les personnes au cours d'un dialogue. Le brillant de la répartie n'est pas le but, davantage l'écoute adulte pour une meilleure compréhension. Le travail, chez nous, est effectué entre personnes qui se respectent à tous les niveaux.

Ces traits se sont transmis dans les différents secteurs de notre entreprise et dans les différents pays où Nestlé opère. En effet, la présence de personnel international, souvent suisse, dans de nombreux marchés a imprimé à tous une manière d'envisager le monde du travail de façon tres homogène.

\section{La notion de qualité}

Pour Nestlé, la qualité est synonyme du nom même de l'entreprise, et certainement l'un des facteurs fondamentaux de son succès ces derniers 125 ans. Pour notre propos, retraçons l'évolution du concept et des systèmes de qualité au cours de ces dernières années, celles que j'ai vécues.

a) Dans les années soixante, le système de contrôle de qualité de notre société était un lourd édifice à trois étages avec des laboratoires de contrôle:

- au niveau de chaque usine le laboratoire de fabrique

- pratiquement dans chaque pays le laboratoire régional

- en Suisse le laboratoire central

Les contrôles étaient multipliés et répétés à chaque étage. L'application stricte des méthodes d'analyses, issues sous forme d'instructions de Suisse, était garantie de la qualité du résultat. Une méthode d'analyse ne pouvait être traduite localement, et les analyses étaient fréquemment répétées sur des échantillons supposés identiques. Le résultat venu de Suisse servant de référencè.

Il n'était à l'époque question que de dire la loi. Je me rappelle avoir arrêté les opérations d'une partie de l'usine où je me trouvais comme chef de laboratoire parce qu'une matière première analysée ne correspondait pas aux normes requises.

b) A la fin des années 60 , alors que le laboratoire régional où je me trouvais couvrait le travail de plusieurs petites unités de production de lait pasteurisé, et dans le but d'uniformiser les systèmes et les méthodes autant que pour former les chefs de laboratoire, je les réunissais, et nous élaborions un système de 'check list' pour l'auto-contrôle des analyses de laboratoire, selon le principe de l'analyse de l'effet de chacune des opérations d'une analyse, pour prendre particulièrement soin des étapes les plus importantes: les chefs de laboratoires de fabrique, au moins, se mettaient à réfléchir.

Au début des années soixante-dix, nous étendions le système aux différents procédés de fabrication des usines du pays où je me trouvais. Prenant une ligne de fabrication dans son ensemble, nous en analysions chaque étape, pour en déterminer l'effet sur le produit, puis décider de la meilleure façon que nous avions de nous rendre compte que l'effet désiré était bien obtenu. Pour ce processus, le chef de laboratoire est 'obligé' de comprendre le produit qu'il contrôle, et de travailler en collaboration avec le chef de fabrication pour établir un système de contrôle qui intègre l'utilisation de données de différentes provenances, analyse d'échantillon d'un laboratoire (et dégustation bien sûr), mais aussi contrôle sur la ligne par l'opérateur de fabrication, données des instruments disponibles sur la ligne, et 'état de maintenance' de la ligne. On peut en dire que c'est un début d'application pragmatique du concept HACCP (hazard analysis of critical control points).

c) Plus tard, à la fin des années septante, dans l'usine où je me trouvais, nous élaborions le système selon lequel, pour être efficace, une information doit être disponible au 'plus bas niveau' capable d'utiliser l'information pour éventuellement effectuer les ajustements nécessaires. Ce qui veut dire:

- que le plus bas niveau doit avoir la formation nécessaire pour comprendre et interpréter le résultat et réagir de façon adéquate,

- que le système d'information doit être efficace pour que l'information arrive à temps là où elle peut être utilisée,

- qu'une atmosphère de confiance existe pour que l'opérateur de fabrication et l'assistant de laboratoire soient suffisamment sûrs l'un de l'autre pour travailler et décider ensemble de changements dans le procédé, dans la pleine confiance de leurs chefs. 


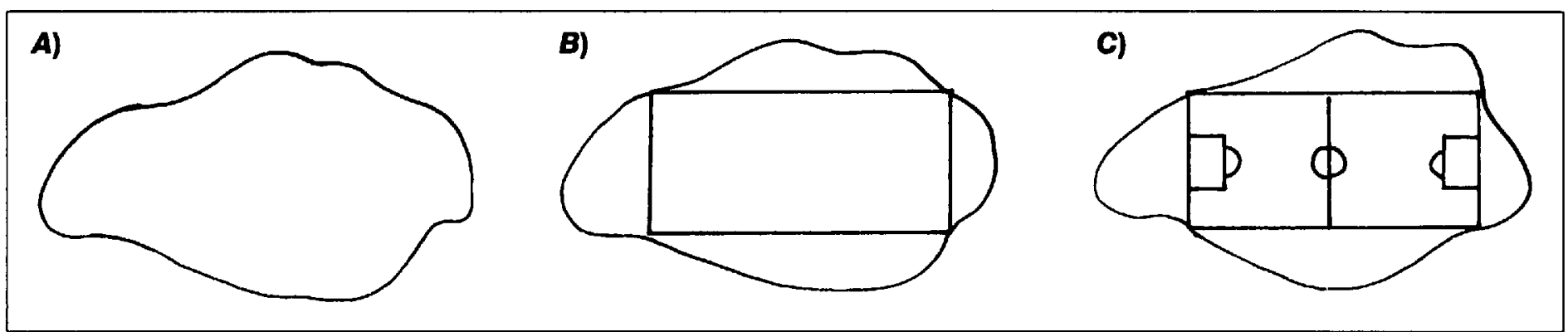

Figure. Les trois dessins

d) Cette organisation se systématise autour du QMS (quality monitoring scheme ou plan de contrôle de qualité) où chaque fabrique établit son propre système de contrôle, intégrant les responsables de la fabrication, de la partie technique et du laboratoire pour l'élaboration, l'application et le suivi d'un document intégré: la qualité est le résultat de la contribution de chacun. Document clef donc car il donne un objectif commun, fixe les responsabilités et établit formellement l'autonomie de chaque établissement dans le cadre d'une philosophie globale. En outre ce document n'est pas statique, car sujet à des révisions fréquentes $=$ il sert de base pour l'analyse d'un problème et s'ajuste pour le résoudre.

Le système est complété par l'autocontrôle au niveau du laboratoire, et la suppression des répétitions d'analyse systématiques aux différents niveaux de laboratoires.

Le QMS cristallise l'analyse qui a été faite des caractéristiques critiques et importantes du produit et du procédé de fabrication appelé HACCP (hazard analysis of critical control points) tout au moins pour la partie opérative, car ce système commence déjà à l'étage de développement du produit.

L'assurance de qualité est ainsi appliquée, sur les bases solides du système de contrôle de qualité, en travaillant de plus en plus en amont pour que le contrôle du produit fini ne serve qu'à vérifier, par un minimum d'indices, que le système d'assurance de qualité fonctionne bien.

\section{Conclusion de la première partie}

Il est bien clair que jusque là, nous n'avons parlé que de produit, alors que la notion de qualité totale englobe bien davantage car elle tend à intégrer toutes les fonctions. Cependant, la même culture baignant toute l'entreprise, les vertus fondamentales dont j'ai parlé plus haut, le goût de l'effort, du travail bien fait, la simplicité des contacts, permettent l'étendue de la notion de qualité du produit à celle de qualité du travail de façon toute naturelle.
Dans son message de fin d'année 1986, notre président, $\mathrm{M}$ Maucher dit à ce sujet: ' $\mathrm{j}$ 'aimerais attirer tout particulièrement l'attention de chacun en 1987 sur l'un des principes fondamentaux de notre politique générale: porter un effort constant sur l'amélioration de la qualité, ce qui signifie non seulement assurer la qualité de nos produits, mais également la qualité dans tous les domaines: les hommes, la gestion, et le travail en général'.

Le concept vient tout naturellement encadrer les développements nécessaires pour suivre l'évolution des affaires.

Par ailleurs, les communications de plus en plus faciles et de plus en plus complètes rendent le travail de chacun plus complémentaire de l'autre chaque jour.

Il y a donc une évolution naturelle, un effort constant de 'coller' mieux aux besoins, une adaptation des systèmes en place.

Cette évolution peut se résumer dans les étapes suivantes:

\section{Inspection}

Récupération

Sélection

Correction

Identification des causes

Contrôle de qualité

Développement de manuels de contrôles

Contrôle des performances du procédé de fabrication

Auto contrôle

Analyse du produit

Planification de certains éléments de qualité

Utilisation de la statistique

Contrôle sur rapports

Assurance de qualité

Libération par des tiers

Audit des systèmes

Planification élaborée de la qualité

Manuels complets

Utilisation des coûts de qualité

Intégration des opérations non productives

Analyse de causes et effets

Contrôle statistique des procédés
Qualité totale

Objectif - amélioration continue

Intégration des fournisseurs et clients

Intégration des opérateurs

Mesure des performances

Travail en équipe

Intégration des employés

On voit que les étapes successives se complètent, on comprend qu'il est difficile de sauter l'une ou l'autre de ces étapes, et que la solidité des résultats de notre compagnie vient justement de cette evolution permanente et de cette recherche d'amélioration dans tous les domaines.

\section{Transition $=$ La discipline}

Ce que nous avons vu jusqu'à maintenant est l'application de systèmes homogènes dans toute l'activité de production de l'entreprise. Pour que ce système donne les résultats escomptés, il faut que la même discipline baigne tous les acteurs dans le système.

Pour que les résultats d'analyse d'un même produit soient comparables, il faut que la même méthode d'analyse soit appliquée partout, exactement de la même façon. Pour que la même sécurité dans la qualité du produit existe, les normes de libération de produit, de communications de défauts doivent être appliquées strictement: on n'interprète pas une norme de libération, on l'applique.

Si bien une conséquence fâcheuse est que le laboratoire devient la police, et certains essaient de voir si leur production passe le contrôle (pas vu - pas pris), on peut être raisonnablement sûr de garder ainsi les défauts à l'intérieur de l'entreprise.

Pour faire comprendre à nos ouvriers rebelles colombiens la nécessité d'une discipline, et surtout la nécessité pour tous de l'accepter, $\mathrm{j}$ 'employais l'explication suivante.

Je demandais que représentait ce des$\sin A$. Devant les regards interrogateurs, je rajoutais quelques éléments $(B)$, puis enfin complétais le dessin $(C)$. Mon explication était simple. Sans régle du jeu, sans limites bien définies, et acceptées par tous, 
il n'y a pas de jeu possible. De plus la règle, ou la limite, ne diminue pas le champ d'action de chaque joueur, mais bien au contraire lui permet d'exprimer ses qualités particulières, d'être plus créatif.

Il m'a semblé utile d'aborder par ce biais le passage à l'aspect que j'estime révolutionnaire de la qualité totale, indiquant par la que loin de permettre à chacun de faire ce qui lui semble le plus opportun, il faut une discipline, ou disons une application des règles du jeu aussi sinon plus stricte. La différence étant qu'elle sera d'autant mieux acceptée qu'elle sera mieux comprise.

\section{Révolution}

D'un système de qualité (inspection, contrôle de qualité, assurance de la qualité), à la notion de qualité totale, la différence que j'appellerai révolutionnaire est dans l'intégration du potentiel, de la capacité et du vouloir des collaborateurs.

Voyons d'abord pourquoi, avant de voir comment et enfin de comprendre certains écueils à éviter.

\section{Pourquoi: \\ D'abord par intérêt.}

Les développements de la technologie ont pour effet de diminuer fortement, voire de supprimer les travaux pénibles ou purement manuels. Un opérateur de fabrication d'une de nos lignes de fabrication de lait en poudre n'a aujourd'hui plus rien à voir avec ce qu'il était il y a dix, voire cinq ans: les systèmes de contrôle intégrés au procédé, les modules logiques de combinaison des éléments de la ligne font appel à la connaissance du produit et des systèmes de contrôle par l'opérateur. La constance de son attention est nécessaire, non plus seulement pour réagir aux déviations de procédé, mais pour en optimiser les éléments. Il recherche la meilleure solution à chaque cas de figure.

Cet opérateur sait avoir la confiance de son encadrement, il communique sa connaissance et son expérience qui devient de plus en plus unique. Ce qui veut dire que l'encadrement s'appuie sur lui (le terme de supervision disparaît); on peut parler d'une complémentarité des fonctions, beaucoup moins de la supériorité de l'une sur l'autre.

Cet apport de la technologie se fait donc en parallèle avec une adaptation organisationnelle. En fait, l'un est indispensable à l'autre. Le chef n'est plus celui qui commande seulement ou celui qui sait le plus seulement. Les organigrammes deviennent plus plats, les communications plus directes; de nouveau le terme de com- plémentarité apparaît, plus celui de réciprocité dans la communication.

La compétitivité vient de l'amélioration de la productivité, une composante importante de celle-ci devenant la contribution de chacun. Cette notion de contribution de chacun se perçoit, et est nécessaire dans tous les domaines: nous avons bien sûr parlé de la production, mais le phénomène s'observe dans la logistique, les services, les ventes, le développement par exemple.

Cette notion de contribution en outre devient de moins en moins perçue seulement fonctonnellement mais intégrée dans un ensemble. J'ai parlé de l'analyste de laboratoire dialogant avec l'opérateur de fabrication; la généralisation dans les services est naturelle pour l'obtention d'une planiflcation optimisée, pour le designd'un nouveau produit, pour la rapidité de la réaction à un événement du marché.

Cette notion de contribution enfin est tournée ver un résultat, donc vers le client, qu'il soit interne ou externe, individuel ou collectif. L'analyse des causes et effets conduit naturellement à l'amélioration de cette perception.

Ce que je viens de traduire comme une nécessité, et ses conséquences logiques est en train de prendre place dans différents secteurs de notre entreprise, de différentes façons et à une vitesse différente d'une fonction, et d'un endroit à l'autre.

\section{Laissez-moi insister sur deux aspects:}

1) On observe de plus en plus, parallèlement à l'organigramme plus plat dont je parlais précédemment, à la perte d'efficacité de ce que j'appellerai le mode de travail de la hiérarchie traditionnelle: l'autorité est de plus en plus conquise par un leader dans une organisation de plus en plus 'personnalisée'. L'organigramme traduit un état d'organisation, il évolue. Dans un cadre où les compétences sont bien définies, et chacun sait ce que l'on attend de lui, le chef donne le support de sa confiance, met à disposition les outils, tant matériels que de formation et d'information, et il coordonne les activités en définissant les objectifs, les cadres de travail, les relations. Il sert en quelque sorte d'arbitre pour le suivi et la gestion, la comparaison des acquis et des différences.

2) Attention au chaos, ce que je clarifierai par cette phrase de Ben, nouvel Eso$p e$ dans ce cas: 'N'importe qui peut avoir une idee'.

De même que la langue est la meilleure des choses chez notre philosophe, il faut donner le support, la confiance à chacun pour qu'il puisse exprimer ses idées et ainsi contribuer aux résultats globaux. Mais il faut garder le contrôle, décider dans les domaines administratifs, résoudre à temps les conflits quand c'est nécessaire. Car attention, de meme que la langue est la pire des choses pour Esope, chacun peut avoir une idée, et l'appliquer, créant le chaos le plus total. La présence du chef, l'organisation et la discipline, sont indispensables pour que l'équipe progresse.

De cette façon, j'aborderai le comment de cette révolution que j'observe.

\section{Comment}

A) Et je voudrais commencer par l'importance du chef. Le leadership est indispensable depuis le sommet de la hiérarchie: La vision du but à atteindre, la communication de cette vision, la constance et la persévérance manifestée tout au long de l'effort, la patience et le courage le long de la route qui n'est jamais sans épines. La confiance manifestée et répétée par le leader appelle en réciprocité la confiance et la motivation de son groupe: Monsieur Maucher, notre président, a défini les qualités qu'il attend de ses chefs de la manière suivante:

'people involvement and Management committment'

'En termes généraux, nous considérons qu'à part leur connaissance professionnelle et leur experience, six caractéristiques sont essentielles pour être un bon leader:

le courage et le sang-froid

la capacité pour apprendre, esprit ouvert, vision

la capacité à communiquer et motiver

la capacité à créer un climat d'innovation penser dans le contexte

la crédibilité: c'est-à-dire, 'cohérence par l'exemple'.

Il est bien certain que la résistance au changement ne disparaîtra pas rapidement, et que chacun recherchera initialement ce qu'il y a de bon pour lui dans le projet de qualité. Mais tout le monde aime à faire partie d'une équipe qui gagne, et c'est le leader qui, au début, en choisissant les objectifs et les communicant à son groupe, en formant ses subordonnés, rappellant constamment les buts et pilotant entre les écueils, forge l'équipe.

$B$ ) Il n'y a pas de recettes universelles ni de système donnant automatiquement les résultats escomptés. D'une fonction à l'autre, d'un endroit à l'autre, les méthodes changent, tenant compte des circons- 
tances historiques, culturelles, des paramètres d'environnement, des résultats acquis.

Une chose est sûre: il ne faut pas copier. Je me rappelle ces entreprises colombiennes clamant à grand renfort d'hymnes d'entreprise et de publicité la naissance de cercles de qualité qui allaient réconcilier l'homme et le travail. Je ne crois pas me tromper beaucoup en affirmant que dans les deux ans, la plupart connaissait une grève dure.

Beaucoup de moyens existent que l'on peut choisir s'ils s'appliquent à l'entreprise et à l'occasion: j'ai là quelques exemples d'usines que je visitais il y a quelque semaines. Les uns travaillent par eux-mêmes, les autres commencent avec des consultants. Certains parlent de cercles de progrès, d'autres de l'établissement d'un système d" "engineering humain'. Dans notre usine colombienne, nous avions appliqué la théorie dite du Feston (une invention locale): au cours des réunions mensuelles d'information, après le passage d'information par le chef, chacun pouvait parler d'un problème qui lui paraissait important (bien sûr que des règles du jeu avaient été établies): on décidait d'une solution, ou de la constitution d'un groupe d'étude (taskforce). Par ailleurs, quelques cercles étaient constitués de façon plus permanente. Ainsi, tout le monde était informé, chacun avait l'opportunité de contribuer à l'amélioration des choses. Il n'y avait pas d'exclusion, et il n'y avait pas de plus et de moins dans le niveau d'intégration (involvement), mais plutôt des aller-retour selon les besoins et les capacités de chacun.

Parfois c'est la notion de zéro défaut qui est la mieux adaptée: souvent maintenant l'occasion de la certification ISO9000 qui n'a rien à voir par elle même avec la notion de qualité totale, mais peut servir de démarreur ou de relais. Nous avions commencé au Danemark une action de ce genre par une analyse, avec la contribution de chaque unité, de nos qualités et défauts (strengths and weaknesses). Un 'marché ami' généralise les essais de 'benchmarking' (mot intraduisible dont la signification est quelque chose comme: comparaison avec les meilleurs dans le but de les égaler).

C) Quelques constantes doivent cependant être relevées.

1) Le succès est seulement obtenu quand la ligne de commandement est utilisée. Il faut donc commencer par l'encadrement pour qu'il soit partie, plus tard de l'implémentation. L'exercice sera probablement plus long, moins spectaculaire, mais beaucoup plus sûr, et l'encadrement en sortira renforcé: c'est-à-dire le passage au leadership sera mieux assuré. Il y a certainement quelque déchets car certains cadres n'ont pas la capacité, ou le désir des changements à obtenir: au moins, si on ne le sait déjà, s'en rendra-t-on compte avant de se trouver dans une impasse, pour amener les correctifs nécessaires.

2) L'information de tous est essentielle, de la façon la plus directe possible. Pour compléter ce que je disais de notre méthode colombienne, je faisais une 'lettre' mensuelle à mes chefs, leur donnant ainsi la substance de ce que je voulais communiquer: il y avait un éditorial et les nouvelles importantes du mois. L'information prend différentes formes: la télévision est parfois utilisée, le simple panneau d'affichage, ce que l'on appelle le dazibao, les systèmes de messageries électroniques. Cen'est pas la technique qui compte, mais le respect de l'interlocuteur à travers une information vraie, claire, compréhensible, renouvelée et intéressante.

3) Une grande attention doit être donnée au besoin de reconnaissance pour le travail bien fait, ou une contribution particulière.

Si on a tous besoin, à des degrés divers, de recevoir la 'tape dans le dos', le signe d'encouragement, 1 'assurance que ce que l'on fait est apprécié, cela ne veut pas dire qu'il faille payer. Ma théorie est que dans l'échelle de Maslow, la reconnaissance par le groupe est plus importante que la securité, et qu'il ne faut donc pas systématiquement 'rabaisser' la contribution en la payant. Mais bien sûr les circonstances changent d'un pays à l'autre et d'une époque à l'autre.

4) Un aspect toujours difficile à optimiser est celui de la formalisation. Si bien j'ai parlé de la nécessité de la règle du jeu, de l'absolue nécessité de la discipline, il faut trouver le moyen d'éviter la bureaucratie par une formalisation excessive.

En effet, c'est une tentation permanente de définir, de légiférer, de régler, dans le but de sécuriser, de 'routiniser', en particulier l'encadrement dans les phases intermédiaires. En bref, il faut se préoccuper des objectifs et résultats à obtenir et donner les limites et les moyens, mais ne pas faire un but en soi de la définition des procédés.

Ce qui compte n'est pas l'efficacité mais l'effectivité, si le mot existe: ce n'est pas de faire bien les choses qui compte, mais de faire bien ce qui est nécessaire pour atteindre le résultat.

\section{Conclusion}

Puis-je, en conclusion, confirmer ce que vous avez préssenti tout au long de cet exposé: nous utilisons avec beaucoup de précaution l'expression de qualité totale.

Il ne faut pas être absolutiste: ne perdons pas de vue que si la qualité est très importante pour nous et constitue certainement une condition nécessaire, elle n'est sûrement pas une condition suffisante. Il faut toujours intégrer les conditions locales, l'environnement économique, les situations historiques, souvent des aspects géopolitiques, sans oublier la concurrence.

Nous avons depuis long temps la conviction basée sur l'expérience que la délégation est le meilleur système dans une organisation telle que la notre: chaque unite définit la date, l'étendue et les moyens des ses projets en fonction des besoins et des circonstances spécifiques. Bien sûr dans le cadre de ses compétences, et d'accord avec notre politique générale définie par notre président par

'Management Committment and People Involvement'

Notre société a maintenant 126 ans d'expérience, et nous avons traversé de nombreuses révolutions, dans le temps et dans l'espace. Cela nous donne:

- cette confiance en nous-mêmes que les étapes suivantes sont à notre portée.

- cette sérénité qui permet de discerner l'accessoire du fondamental et d'aller à pas comptés mais sûrs.

- cette vision du long terme sans laquelle rien de solide ne se construit, et qui renforce la persévérance et la patience.

Evolution ou révolution: cela n'est pas le dilemme: dans un monde qui change chaque jour, et chaque jour plus vite, c'est seulement grâce à la contribution de tous que nous irons de l'avant.

De cela oui, nous sommes sûrs: nos 126 ans de succès sont dus à la contribution des collaborateurs qui se sont succédés dans l'entreprise. Et c'est notre responsabilité, aujourd'hui, de former ceux qui guarantiront la perénité et forgeront les succès de demain. Il nous faut donc, jour après jour, tout mettre en œuvre pour que:

- tous les cadres de l'entreprise

- se sentent investis de la responsabilité particulière

- de mobiliser toutes les ressources de l'entreprise,

- dans un effort constant d'amelioration,

- et que leur action soit mobilisatrice de leur personnel

- à qui ils donnent les éléments de connaissance et de responsabilisation nécessaires

- pour que leur contribution soit à la mesure de leur potentiel. 